\title{
Transformation und Transparenz: Warum im Journalismus das "Quellen-W « wichtiger wird
}

\section{Sebastian Köhler}

Keywords: Journalismus, Quellenangabe, Transparenz, Vertrauenskrise, Transformation, W-Fragen, Quellen-W, Völkerverständigung, friedliche Koexistenz

\section{Abstract}

Der Beitrag diskutiert in verschiedener Hinsicht die Wichtigkeit möglichst frühzeitiger Quellenangaben in journalistischen Beiträgen. Hierbei geht es um zentrale Aspekte von Transparenz im Journalismus, auch im Kontext der Befunde zu Misstrauen gegenüber etablierten Medien. Repräsentative Befragungen, öffentliche Diskussionen um Medienqualität und auch Erfahrungen aus eigener journalistischer Arbeit weisen darauf hin, dass Quellentransparenz als (zunehmend) wichtiger Faktor für Vertrauen gegenüber journalistischen Angeboten diskutiert werden sollte. Auch im Hinblick auf zwischenstaatliche Spannungen, Krisen und Konflikte erscheint das Konzept essenziell. Der Beitrag zeigt anhand der Berichterstattung zu den Fällen Babtschenko, Skripal und Nawalny, dass durch mangelhafte Quellentransparenz Spannungen in der Tendenz geschürt werden und sorgfältigen Quellenangaben ein Transformationspotenzial in Richtung friedlicher Koexistenz innewohnt.

Für Hinweise zu einer früheren Fassung dieses Beitrages danke ich Michael Haller, Uwe Krüger und Kerem Schamberger.

Sebastian Köhler: Transformation und Transparenz: Warum im Journalismus das »Quellen-W" wichtiger wird. In: Nils S. Borchers, Selma Güney, Uwe Krüger und Kerem Schamberger (Hrsg.): Transformation der Medien - Medien der Transformation. Verhandlungen des Netzwerks Kritische Kommunikationswissenschaft. Frankfurt am Main: Westend 2021. DOI: https://doi.org/10.53291/ERWL7086. 


\section{Einleitung: In Richtung gelingender gesellschaftlicher Kommunikation}

Most information circulating is doing so precisely because those responsible for it want people to know about it [...] If governments and business are to be held to account, as more than

ever they need to be, then democracy requires a functioning, independent news media. A move towards greater transparency in sourcing might be a step in that direction.

(Phillips 2010, 1, 8)

Das Thema dieses Beitrages lautet: Transformation und Transparenz. Denn Journalist*innen sollten sich angesichts einer anhaltenden Vertrauenskrise (zum Strukturwandel öffentlichen Vertrauens vgl. Haller 2017) gegenüber ihrem Berufsfeld und angesichts wachsender sozialer Spannungen in Gesellschaften wie der in Deutschland nicht zuletzt um mehr Transparenz ihrer Arbeit bemühen (siehe Köhler 2018; Köhler 2019, 76-78). Auch laut der Mainzer Langzeitstudie Medienvertrauen von 2019 war der Anteil derjenigen Personen in Deutschland, die etablierten Medien gegenüber skeptisch sind (die Studie nennt sie sogar »Medienzyniker«; Schultz et al. 2020, 322), zumindest »nicht marginal«. Etwa jede Vierte vertraue etablierten Medien nicht (mehr) bei wichtigen Themen (ebd., 329): „Geht es um politisch besonders umstrittene Fragen, die in aufgeheizter Atmosphäre diskutiert werden, sind die Vertrauenswerte noch geringer.« Dabei handelt es sich um etwa doppelt bis dreimal so viele Menschen wie jene, die 2017 bei der Bundestagswahl die AfD gewählt hatten (12,6 Prozent), was offenbar auf ein tiefergreifendes gesellschaftliches Problem verweist: Misstrauen gegenüber den Medien ist kein Phänomen, das nur den rechten Rand betrifft.

Dabei zählt gerade Quellentransparenz seit Jahrzehnten zum »Kernbestand « vieler journalistischer Qualitätskataloge (Schultz 2019), was auch Silvio Waisbord mit Blick auf digitalen und tradierten Journalismus unterstreicht: Redaktionelles Selbstverständnis bezieht sich weiterhin auch auf Transparenz nicht nur im Sinne von Quellentransparenz als journalistische Norm: »A blend of modernist values (truth, transparency, rationality, factivity, freedom) still anchors the collec- 
tive imaginary of newsrooms.« (Waisbord 2019, 356) Entsprechende Aspekte und Ebenen insbesondere öffentlicher Selbsttransparenz im Journalismus (vgl. Reimer 2017) diskutiere ich in Abschnitt 3.

Schließlich können, ja, sollen journalistische Medien hinsichtlich sozialer Transformationsprozesse und des Aufhebens globaler Krisentendenzen und Pathologien (vgl. Rosa 2013, 89-91; Krüger und Meyen 2018) wesentliche Rollen spielen. Hans-Peter Krüger hat Entsprechendes im Aufgreifen von Vorschlägen Bertolt Brechts und Juri Lotmans als Beiträge zur kulturellen Aufgabe öffentlicher Medien in modernen (oder spätmodernen) Demokratien bestimmt (Krüger 1992, 220-222). Diese gleichsam transformative kulturelle Aufgabe (die auch Politik und Wirtschaft verändern dürfte) kann als »Prinzip Perspektivenwechsel« begriffen werden: Indem man Sichtweisen aufzeigt und sie als solche explizit kenntlich und damit kritisierbar macht, ermöglicht und erleichtert man tendenziell Wechsel. Diese sollten möglichst symmetrisch, auf Augenhöhe erfolgen. Denn im Zuge der Digitalisierung gilt entsprechendes »technisches Kapital« (Rudolph 2019, 104-105) als wichtige Zugangsmöglichkeit zu Information und Kommunikation sowie als entscheidende Ressource in einer Wissensgesellschaft. »Technisches Kapital meint hier im Fortschreiben der Kapitalbegriffe von Marx und Bourdieu, dass sowohl der infrastrukturelle Zugang zum Internet als auch dessen praktisch-technischer Gebrauch durch die sozialen Positionen der Akteur*innen bestimmt werden. Wenn es ungleich oder ungerecht verteilt ist oder wird, dann sorgt die »digital divide« für weiter wachsende soziale Spannungen und Spaltungen.

Was bedeutet das mit Blick auf Quellentransparenz? Ein (normativer) Bereich prinzipiell Perspektiven wechselnder, möglichst symmetrischer Kommunikation mag (gleichsam als Mesoebene) zwischen »Mikro« (individuellem journalistischem Handeln) und »Makro« (Öffentlichkeiten) vermitteln. Diese Verbindung kann auch auf neue Weise als redaktionelles Vermitteln bestimmt werden, sofern Redaktionen nicht als »Sender«, sondern als offen Beitragende begriffen werden (vgl. Köhler 2018). Als Organisationen journalistischen Handelns lassen sich diese hier auf neue Weise als Bindeglied zwischen Individuen (Mikroebene) und dem sozialen Bereich Journalismus (Makroebene) modellieren.

Nutzer*innen ihrerseits sollten daher die Angebote besser einordnen und Rückmeldung geben können. Angela Phillips (2010, 2) schreibt: 》Citizens are more easily able to trace information to its source.« Journalist*innen wiederum dürfte diese Art von Vermittlung darin bestär- 
ken, möglichst professionell, selbstkritisch und vielfältig zu arbeiten, hier insbesondere reflektiert und innovativ (auch »gegen den Strom«) Quellen auszuwählen sowie diese dann - kritikoffen - am besten von vornherein in journalistische Beiträge einzubeziehen. Damit würde im Idealfall die kommunikative Kompetenz tendenziell aller Beteiligten gewissermaßen als Vermittlungsleistung wachsen. »Kritikoffen« soll hier unterstreichen, dass Nutzer*innen die Quellen nicht nur kennen, sondern sowohl sie als auch deren Auswahl durch die Redaktion einfacher kritisieren können.

In dieser Richtung mögen die sich historisch entwickelnden oder wandelnden Alltags- und Expert*innenkulturen als gleichrangig gelten-Minderheiten können recht haben und bisherige Hierarchien (zum Beispiel die zwischen Sender*innen und Empfänger*innen) in Frage gestellt werden. Solche Offenheit dürfte einen Wertewandel befördern, der zu neuen Mehrheiten, jenseits tradierter Markt- oder Machtdominanzen, zu führen vermag. Es geht um eine Symmetrisierung von Perspektiven in gesellschaftlicher Kommunikation. Dafür scheint wechselseitiges Vertrauen ein wichtiger Aspekt, sowohl der Journalist*innen in ihre Publika als auch der Nutzer*innen in die journalistischen Angebote. Wenn wir daher auch Kommunikation als das stets erneute Durchlaufen der Differenzen zwischen Kommunikablem und Nichtkommunikablem begreifen, dann wird klar: Die gesamtgesellschaftlich möglichst gleichberechtigte Produktion und Reproduktion dieser Differenzen führt tendenziell zur Entdeckung neuer Themen und Lösungsmöglichkeiten, die bisher keine (oder kaum eine) Rolle spielten. So kann es, was die Transformationsproblematik in Richtung nachhaltiger sozial-ökologischer Demokratisierung angeht, zu grundlegenden Umorientierungen der gesellschaftlichen Kommunikation kommen (vgl. Krüger 1992, 228).

Das heißt für den Journalismus unter anderem: Das Nutzervertrauen hängt nicht zuletzt ab von journalistischer Transparenz, sowohl bezogen auf die Arbeitsprozesse (Themenwahl, Recherche, Produktion, Distribution und redaktionelle Selbstkritik) als auch auf die manifesten Beiträge selbst. Studien deuten darauf hin (vgl. exemplarisch Prochazka 2020, 188), dass »Transparenz « (hier als eines von 19 Qualitätsitems abgefragt) in dreierlei Hinsicht als problematisch gelten kann: Bei der Größe der Diskrepanz zwischen der Wichtigkeit des Qualitätskriteriums und der wahrgenommenen Einlösung beziehungsweise Umsetzung durch die Medien liegt »Transparenz« im Vorderfeld (Rang 
6 von 19). Zudem sind hier laut Studie die Erwartungen der Nutzer*innen extrem gering (Rang 2).

Bisher war es eher die fünfte (und damit meist letzte) der sogenannten W-Fragen, die eine Nachricht als Meldung oder Bericht prägen sollen: Welche Quellen werden ausgewählt und welchen Quellen wird (inwiefern) vertraut? Gerade diese Fragen scheinen mit Blick auf die massive Zunahme journalistischer Angebote einerseits und die deutlichen sozialen und nicht zuletzt medienbezogenen Segregationstendenzen andererseits an Dringlichkeit zu gewinnen, insbesondere für das normative Ziel gelingender gesellschaftlicher Kommunikation im Sinne von Jürgen Habermas oder Michael Haller (Köhler 2015, 13-14). Dieses lässt sich auch beschreiben als eine möglichst rückkopplungsreiche Gesellschaft wie bei Dietmar Dath und Barbara Kirchner (vgl. ebd.) oder als eine Gesellschaft responsiver, resonanter Wechselverhältnisse »Zwischen Selbst und Welt« (Rosa 2013, 147).

\section{Einige Beispiele aus der Krisenberichterstattung}

Fehlende Quellentransparenz scheint ein grundsätzliches Problem im gegenwärtigen Journalismus zu sein, was angesichts von kontrovers bewerteten Krisenlagen seit etwa 2010 vielleicht deutlicher wird als zuvor. Zwei kurze Beispiele zunächst mit Blick auf die höchst umstrittene Situation in Bolivien 2019 im Zusammenhang mit der Absetzung von Evo Morales, die wie auch der folgende Fall Babtschenko für mediale Umgänge mit der transformationstheoretisch und -praktisch grundlegenden Frage »Krieg oder Frieden? - oder konkret: »Internationale Eskalation oder Entspannungsbemühungen?«- stehen. ${ }^{1}$

a. »Das Militär will die Ordnung wiederherstellen«, meldete etwa die Süddeutsche Zeitung online am 12.11.2019 (Gurk 2019). Man rieb

1 In Kapitel 1, Artikel 1 der UN-Charta heißt es seit 1945: „Die Vereinten Nationen setzen sich folgende Ziele: 1. den Weltfrieden und die internationale Sicherheit zu wahren und zu diesem Zweck wirksame Kollektivmaßnahmen zu treffen, um Bedrohungen des Friedens zu verhüten und zu beseitigen, Angriffshandlungen und andere Friedensbrüche zu unterdrücken und internationale Streitigkeiten oder Situationen, die zu einem Friedensbruch führen könnten, durch friedliche Mittel nach den Grundsätzen der Gerechtigkeit und des Völkerrechts zu bereinigen oder beizulegen.« https://www.unric.org/de/charta. Zugegriffen: 16. November 2019. 
sich die Augen: Welche Ordnung? Und warum gerade das mit Putschvorwürfen konfrontierte Militär? Wieso wird der Satz als Tatsache ohne Quellenangabe behauptet? Das Mindeste wäre, die Version - sofern es sich nicht um (recherchiert) »Unstrittiges« wie »Heute ist Sonntag « handelt - als Version (vgl. Haller 2008, 60; siehe auch Schultz 2019 mit Bezug auf journalistische Quellenkritik) kenntlich zu machen, in etwa also: »Militärführung: Wollen Ordnung wiederherstellen«.

b. Im ARD-Teletext hieß es am 13.11.2019 um 21.10 Uhr auf Tafel 112: »Bolivien hat Interimspräsidentin«. So werden nicht zuletzt medial »Tatsachen « geschaffen. Die rechtsgerichtete Senatorin Jeanine Áñez hatte sich selbst zur Übergangsregentin ernannt, ohne jede nachvollziehbare demokratisch-parlamentarische Legitimation. Auch hier wäre eine zumindest implizite Quellenangabe hilfreich zum Verständnis, im Sinne von »Senatorin ernennt sich zur Interimspräsidentin « - allemal nachrichtlich treffender als die zitierte Überschrift.

Phänomenologisch-explorativ möchte ich zum umfassenden Aufschließen dieses Problemfeldes ausgehen vom Fall zahlreicher Meldungen über die vermeintliche Ermordung des russischen Journalisten Arkadi Babtschenko im Jahr 2018. Eine Überschrift wie »Ukrainische Behörden: Babtschenko getötet « kann in diesem Kontext als empirisch wahre Aussage gelten. Leider hatten sich an jenem 29. Mai nur sehr wenige Medien so geäußert. Stattdessen texteten die Vertreter*innen direkt im Sinne von »Babtschenko getötet« oder »Babtschenko ermordet«. Dass man sich auf Behörden in Kiew als Quelle bezog, tauchte, wenn überhaupt, erst im Laufe des Beitrages auf. Der Fall entpuppte sich wenig später als ein Manöver des ukrainischen Geheimdienstes SBU.

Mein Beitrag soll auch an dieser Stelle ein Plädoyer für (mehr) Transparenz im Journalismus sein, nicht zuletzt mit Blick auf die Quellenlage. Und deshalb ein pragmatischer Vorschlag vorab: Nennen wir doch die (wichtigste oder eben einzige) Quelle so früh wie (sinnvoll und angemessen) möglich. Das sollte es den Nutzer*innen von vornherein und gleichberechtigt ermöglichen, den Beitrag einordnen zu können. Im Fall Babtschenko ruderten viele Medien am 30. Mai 2018 nach Bekanntwerden des Fakes zurück. Sie übten allerdings auch dabei kaum Selbstkritik. Stattdessen wälzten sie die Schuld für dieses spezielle journalistische Versagen nun wahlweise auf Leute aus der Ukraine oder aus Russland ab. 
Abb. 1: Meldung in der 17-Uhr-

Tagesschau am 29.05.2018 im

$A R D$.

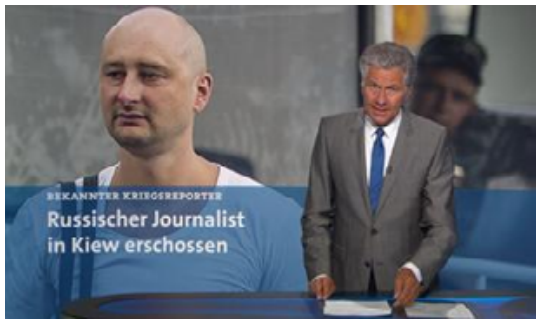

UPDATE $30.05 .2018,15: 26$ Uhr

\section{Der Mord an Arkadi Babtschenko verdeutlicht die russisch-ukrainische Feindschaft}

Der Journalist Arkadi Babtschenko wird vor seiner Wohnung in der ukrainischen Hauptstadt Kiew erschossen. Der Vorfall sorgt für weitere Spannung zwischen Kiew und Moskau. voN OLIVER BLger

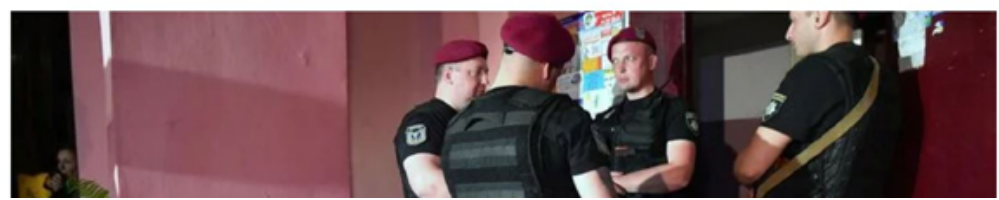

Abb. 2: Schlagzeilen ohne Quellenverweis im Tagesspiegel (Bilger 2018).

Daher nun als phänomenologischer Aufschluss einige medienkritische Aspekte zum Fall Babtschenko, exemplarisch anhand eines längeren Beitrages auf Spiegel Online (vks/dpa 2018). Dieser stammt nach eigenen Angaben der Redaktion vom 1. Juni 2018 um 18.37 Uhr, erschien also etwa zwei Tage, nachdem die ersten Beiträge zum Thema veröffentlicht worden waren. Die Quelle »Ukrainische Behörden« wurde in vielen etablierten deutschen Medien nicht oder kaum angegeben, wie zum Beispiel in der 17-Uhr-Tagesschau am 29. Mai (Abb. 1). Weder in der Dach- noch in der Schlagzeile benennt man die Quelle. Dabei wäre durchaus Platz gewesen - »bekannter Kriegsreporter « und »russischer Journalist« wirken hier synonym. Ähnlich sah das am 30. Mai im Tagesspiegel aus (Abb. 2).

Silvio Waisbord erklärt zu Phänomenen wie diesem mit Blick auf Entwicklungen im tradierten und im digitalen Journalismus: »Readers consume absolute fictions as if they were (f)actual representations of reality, and engage with news without much concern for the identity or the legitimacy of the source.« (Waisbord 2019, 357) Und dies könne auch so interpretiert und kritisiert werden: »Rogue actors spread false information.« (ebd.) Andere Nutzer*innen mögen sich abwenden oder 
den Beiträgen erst gar nicht Aufmerksamkeit schenken, weil sie sich von solchen intransparenten Einseitigkeiten kaum angesprochen fühlen.

Bemerkenswert hierbei eine offenbar durch den digitalen Journalismus und dessen Dynamisierungen tendenziell beförderte Verschiebung mit Blick auf die Quellenlage: Im Fall Babtschenko haben viele Journalist*innen und Redaktionen wie naive Konsument*innen gewirkt: Sie zeigten bemerkenswert wenig Sorge hinsichtlich Identität oder Legitimität der Quelle. Die Herkunft wurde anscheinend nicht nur kaum geprüft, sondern darüber hinaus auch nur selten (und dann eher durch Nischenmedien) von vornherein angegeben.

Einen Erklärungsversuch für das Erheben einer offenbar interessierten Version, einer Fälschung, in den Rang einer Tatsache deutet der Spiegel-Online-Beitrag gegen Ende an: »Viele Journalisten [hier müsste man sagen: prowestliche Journalist*innen; SK] trauerten am Tag des angeblichen Mordes « - und waren offenbar kaum zur professionellen Nachrichtenproduktion geschweige denn zur Analyse fähig. Es ging vermeintlich ganz klar um »den Mord an dem erklärten Kremlkritiker«. Als Kronzeugen zitiert Spiegel Online einen erklärten Freund Babtschenkos, den Investigativreporter Pawel Kanygin von der Moskauer Nowaja Gaseta: »Das ist ein Terroranschlag auf die Gemeinschaft von Journalisten in Russland und in der Ukraine.« Der Kollege sei dann verständlicherweise erleichtert gewesen, als die Fälschung bekannt wurde: »Er lebt, das ist das Wichtigste! Und abends kriegt er eins hinter die Löffel, weil mir die letzten Haare ausgegangen sind.«

Doch auch dieser Beitrag kommt zumindest zu dem (offenbar kritisch gemeinten) Schluss: »Die Glaubwürdigkeit der Medien leidet unter solcher Irreführung «, und »Journalisten müssen noch intensiver und noch viel genauer hingucken«, zitiert der Spiegel-Online-Beitrag wiederum Frank Überall, den Vorsitzenden des Deutschen Journalisten-Verbands (DJV). Das ist nicht falsch, aber oft würde es - angesichts (leider) geringer und weiter schwindender Ressourcen für journalistische Arbeit - schon helfen, professionelle Standards der Nachrichten(Re-)Produktion zumindest zu halten, oder eben dialektisch aufzuheben. Hier würde das bedeuten, die Quelle ganz sachlich zumindest klar und von vornherein anzugeben, falls die Ressourcen nicht für weitergehende Recherchen reichen, und sich nicht von Emotionen hin- und wegreißen zu lassen.

Zu guter Letzt kommt Pawel Gussew, Chefredakteur der russischen Zeitung Moskowski Komsomolez, zu Wort (ebd.): »Das ist nicht nur eine Provokation gegen Russland. Das ist auch eine Provokation Babtschen- 
kos gegen die ganze Journalistenzunft.« Beides ist nicht von der Hand zu weisen. Und in beide Richtungen, bezüglich Länder wie Russland, aber auch hinsichtlich des Journalismus, mag mit Blick auf zu verbessernde Quellentransparenz aus Fällen wie diesem gelernt werden. ${ }^{2}$ Es dürfte (auch hier) Transformationsbedarf bestehen: Für den Journalismus, für die gesellschaftliche Kommunikation und für neue Entspannungspolitiken in einer multipolaren Welt.

\section{Systematisches zu Transparenz und Quellen-W}

Nun seien hier einige wichtige Aspekte zur Entwicklung der Rolle des Quellen-W in Rhetorik und Journalismus diskutiert. Denn auch in deren Verlauf gab es Transformationen, die zu Wieder-Entdeckungen im Sinne dialektischen Aufhebens einladen.

»Transparenz « zählt seit langem zu den Kernforderungen an journalistische Qualität (Reimer 2017, 101). Sie soll einerseits und traditionell die Gegenstände journalistischer Beiträge durchschaubar(-er) machen, aber als gewissermaßen reflexive Qualität auch Bedingungen und Prozesse journalistischen Arbeitens selbst erkennbar und damit (besser) kritisierbar werden lassen.

Hier ist vor allem der zweite Aspekt interessant. Zunächst sei diese journalistisch-reflexive Transparenz die Gesamtheit jener journalistischen Kommunikationen, die nicht zuletzt an Rezipienten gerichtet sind und über die journalistische Aussagenproduktion sowie ihre Rahmenbedingungen informieren sollen (Reimer 2017, 102-103). Beim Schaffen journalistischer Selbsttransparenz lassen sich drei Dimensionen unterscheiden (ebd., 103-104):

1. In der ersten Dimension kann zwischen einer Objekt- und einer Metaebene unterschieden werden, auf denen Journalist*innen Transparenz herstellen: a) das Produkt, also der einzelne journa-

2 Es ist kein Einzelfall: Ich habe an anderer Stelle (Köhler 2019, 73-98) ähnliche Medienphänomene mit Blick auf den »Fall Skripal« ausführlich diskutiert. Im Jahr 2020 wiederum stand schon früh für viele Medien hierzulande fest und wurde als Tatsache vermeldet: »Nawalny vergiftet«, und zwar unter Schlagzeilen wie »Konflikt mit Russland«. Quellen wie »Bundesregierung« oder »Bundeswehrlabor« wurden nicht oder kaum angegeben; beispielsweise ZDF: »Nawalny vergiftet«, https://www.zdf.de/nachrichten/video/politik-nawalny-vergiftungnervenkampfstoff-100.html. Zugegriffen: 31. Oktober 2020. 
listische Beitrag, und b) der Prozess, also redaktionelle Routinen und Entscheidungen.

a. Produkt- beziehungsweise Beitragstransparenz soll dem Publikum ermöglichen, auf den Beitrag zu reagieren, weil Verschiedenes überprüft werden kann, was der intersubjektiven Nachvollziehbarkeit in der Wissenschaft ähnelt. Und genau hier ist Quellentransparenz das wohl wichtigste Beispiel: Journalistisch Tätige sollten sowohl die Quelle(-n) explizit (oder zumindest implizit) nennen als auch bei deren Einordnung helfen, indem nicht zuletzt die dahinterstehenden Interessen deutlich gemacht werden.

b. Prozess- beziehungsweise redaktionelle Transparenz hingegen soll auf Entscheidungskontingenzen der redaktionellen Ebene sowie auf entsprechende Rahmenbedingungen hinweisen. Beispiele wären hier die Begründung von Themenauswahl und -umsetzung, die Erläuterung redaktioneller Richtlinien oder auch die Offenlegung von persönlichen Interessen und Interessenkonflikten. »Actor transparency « (ebd., 104) soll den Mediennutzer*innen helfen, Journalist*innen als Personen (besser) einzuordnen, zum Beispiel hinsichtlich deren politischer oder sonstiger soziokultureller Orientierung. Laut Reimer lassen sich diese beiden Arten von Selbsttransparenz zwar unterscheiden, aber kaum trennen: Enthält ein Beitrag einen Fehler, wird er häufig nicht nur verbessert (Beitragstransparenz), sondern es wird auch erläutert, wie und warum es im journalistischen Prozess zu diesem Fehler kommen konnte (redaktionelle Transparenz).

2. In einer zweiten Dimension zerfällt die Transparenz Reimer zufolge nach Kommunikationsform in monologische (Übertragung der Redaktionskonferenz, Angabe von URLs) und dialogische Transparenz (Chat mit Nutzer*innen).

3. In einer dritten Dimension kann differenziert werden, ob das Transparenz-Instrument auf das Internet angewiesen ist oder nicht.

Eine weitere Ebene der Transparenz wäre eine Unterscheidung nach Grad des Nutzens für die Publika als Kommunikationstransparenz: Was ist aus Sicht der Publika notwendig (»must have«), was ist eine sinnvolle Ergänzung (»nice to have«) und was stört sogar eher bei der Nutzung? ${ }^{3}$ Gerade dafür wäre mehr Austausch auf Augenhöhe mit Me-

3 Sicher erhöhen zusätzliche Transparenz-Informationen in der Tendenz die 
diennutzer*innen sinnvoll - zum Beispiel zu Fragen des Einblendens (Insertierens) von Quellen in nachrichtlichen Videobeiträgen. Denn es gibt Hinweise, dass etablierte Redaktionen (wie ARD-Aktuell) den Bedarf zumindest von Teilen ihrer Publika nach Quellentransparenz eher unterschätzen (ebd., 106). Dabei lassen sich neben dem Material, das dort selbst produziert wurde, mindestens sechs Quellenarten unterscheiden: 1) kommerzielle Quellen (PR-Material von Konzernen), 2) andere organisationsnahe Quellen (PR-Material von Greenpeace oder Sportclubs), 3) (quasi-)staatliche Quellen (Material von Regierungen oder Behörden), 4) user-generated content (Nutzerinhalte vor allem via Smartphones), 5) journalismusähnliche Quellen (journalistisch orientierte Blogger wie Rezo oder Rayk Anders) und 6) tradiert-journalistische Quellen (andere Redaktionen, die auch nach professionellen Maßstäben arbeiten, zum Beispiel Agenturen wie AP, Reuters und AFP oder Sender wie CBS, BBC und ZDF).

Nun zu einigen Aspekten der Quellenfrage: In Journalismus und Journalistik soll »Quelle« das Material bezeichnen, das einem journalistischen Beitrag zugrunde liegt (Schultz 2019). Daher gehe es zugleich um dessen Herkunft: Eine Quelle sei diejenige Person, Institution oder Kommunikation, von der eine bestimmte Information herrühre. Viele Arten von Quellen haben sich über die Jahrhunderte mindestens ebenso professionalisiert wie der Journalismus - Medienprofis der Auftragskommunikation in Wirtschaft, Politik, NGOs und Vereinen wissen, wie sie redaktionelle Aufmerksamkeit induzieren. Das sollte für den Journalismus gerade hinsichtlich der Quellentransparenz als Herausforderung verstanden werden: Denn vieles an gesellschaftlich wirksamen Informationen ist ja gerade deshalb in der Welt, weil es an deren Zirkulieren ein (oft machtvoll organisiertes) Interesse gibt, wie etwa Angela Phillips (2010) unterstreicht.

Im digitalen Journalismus kann praktisch jeder zur Quelle werden. Auch Silvio Waisbord (2019, 352-354) zufolge wird nicht mehr (nur) zur Nachricht, was ausschließlich Journalismus-Profis als (einstige) Gatekeeper in ihren Redaktionen entscheiden. Neben »ordinary citizens« fallen hier vor allem (nicht so ganz) neue professionelle Ak-

Komplexität journalistischer Medienangebote - das kann laut Reimer auf Kosten der Verständlichkeit gehen und die Aufmerksamkeit des Publikums von der eigentlichen Nachricht ablenken (Reimer 2017, 109). Betriebswirtschaftlichökonomistisch Orientierte mögen monieren, es handle sich bei besserer Quellen-Transparenz um »Verschwendung von Zeit, Aufwand, Personal und anderen Ressourcen« (ebd.). 
teur*innen als Quellen auf: »Public relations and marketing firms have perfected the science of news virality and native advertising. Government agencies flood the Internet with content that resembles traditional news.« (ebd.) Die Verhältnisse dürften eher komplizierter werden: "News authorship is also more complicated as networks of authors permanently produce layers of content by adding, discussing, excising, deleting, and reframing content.« (ebd.) Die Interessen der Quellen sind ein wichtiger Aspekt für die journalistische Arbeit: »Multiple actors have many motivations to participate in news-making [...] Political propagandists spread news to deceive voters. Legislation mandates government agencies to disseminate news and information.« (ebd.) Gerade weil Organisationen offenbar auch PR betreiben, die nicht mehr selektive Tatsachen verbreitet, sondern anscheinend zielgerichtet Falsches vermittelt, sollte es also im Journalismus wichtiger werden, die Quellen dafür einerseits zu prüfen und andererseits im jeweiligen Beitrag frühestmöglich deutlich zu machen. Denn ein »vorgelagerter Faktor« für das Vertrauen der Publika in journalistische Beiträge dürfte das Vertrauen von Journalist*innen in Quellen sein (Krüger 2020). An Bedeutung scheinen dabei »distanzierte Quellen« zu gewinnen, also Akteur*innen, welche die Journalist*innen nicht persönlich getroffen haben, sondern deren Angebote sie oft über Internetplattformen von Intermediären (Soziale Medien wie Twitter) erreichen - nicht zuletzt in der Krisen- oder Konfliktberichterstattung (vgl. Wintterlin 2019).

\section{Normativ-pragmatische Aspekte als Ausblick - Ansätze für Transformationen über den Journalismus hinaus}

Quellentransparenz (selbstverständlich unter Berücksichtigung etwaigen Quellenschutzes bei investigativen Beiträgen) als ein wichtiger Aspekt von Selbsttransparenz kann als notwendiges Kriterium für journalistische Beiträge gelten. Natürlich lassen sich auch andere Perspektiven relativ »fair« ganz ohne Quellenangabe darstellen. Pragmatistisch mag man an der Stelle darauf hinweisen, die Mediennutzer*innen nicht durch zu viele oder zu frühe Quellenangaben zu überfordern. Damit aber vergäbe man durch Über-Vereinfachung Potenziale für besser gelingende gemeinschaftliche und gesellschaftliche Kommunikation, die hier durch leichtere Perspektivenwechsel und möglichst symmetrische Kom- 
munikation vermittelt wird. ${ }^{4}$ Nicht nur Angela Phillips (2010, 8; 2011, 58; vgl. Reimer 2017, 108) argumentiert daher, dass es - sofern sich mehr und bessere Quellentransparenz als professionsweite Norm durchsetze - für Journalist*innen schwerer werde, besonders einseitig (und gleichsam unbemerkt) Quellen zu verwenden und so tendenziell Fakten sogar zu verdrehen. Andere nennen damit Verwandtes auch das Phänomen »opportuner Zeugen « (Schultz 2019), also das einerseits gezielte und dann andererseits oft auch intransparente Zitieren solcher Quellen, die der (politischen) Tendenz eines Beitrags, eines journalistisch tätigen Individuums oder einer Redaktion am meisten entsprechen.

In Deutschland ist die Rechtslage, was die Sorgfalt im Umgang mit der Herkunft von Ausgangsdaten angeht, durch die Landespressegesetze bestimmt, die sich alle recht ähnlich zu dieser Frage verhalten. Hier sei stellvertretend auf das Berliner Landespressegesetz verwiesen. ${ }^{5}$ In Paragraph 3 heißt es dort zur öffentlichen Aufgabe der Presse und damit der journalistischen Medien überhaupt in Absatz 2: „Die Presse hat alle Nachrichten vor ihrer Verbreitung mit der nach den Umständen gebotenen Sorgfalt auf Inhalt, Wahrheit und Herkunft zu prüfen.« Diese Prüfung sollte wie seit Jahrzehnten gefordert sicher weiterhin geleistet werden, aber der Fall Babtschenko macht auch deutlich: Es bleibt ein systematisches Restrisiko, dass sich die Ausgangsinformationen (»Babtschenko getötet«) schlicht als nicht wahr erweisen - egal, ob nun bewusst gefälscht (manche nennen das dann »Fake News«) oder eher zufällig falsch. Deswegen gehört zur professionellen Sorgfalt beim prüfenden Umgang mit Ausgangsdaten, dass auch und gerade für die Nutzer*innen die Herkunft des Materials möglichst sofort erkennbar und nachvollziehbar werden sollte. Solches Herangehen dürfte die

4 Die Organisation The Media Standards Trust hat in London das Meta-Tag »hNews« für Mikroformate entwickelt, das interessierten Nutzer*innen zeigen soll, wo die Information zuerst auftauchte, was ihre weitere Publikationsgeschichte war und welche Rahmenbedingungen auf den entsprechenden Plattformen galten. Ähnliche Tags wurden auch von der New York Times und anderswo entwickelt (Garton Ash 2016, 188). Diese Daten sollten auch nicht als störend erscheinen: »It is visible only for those who want to access it.« (Phillips 2010, 7)

5 Berliner Pressegesetz vom 15. Juni 1965: http://gesetze.berlin.de/jportal/ portal/t/173q/page/bsbeprod.psml;jsessionid=FEC85A598D974D2282499A6 A0CA8D862.jp29?pid = Dokumentanzeige\&showdoccase $=1 \&$ js_peid $=$ Trefferliste\&documentnumber $=1 \&$ numberofresults $=28 \&$ fromdoctodoc $=$ yes $\&$ doc .

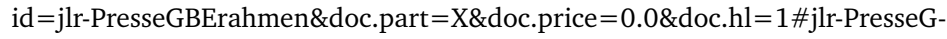
BEpP3. Zugegriffen: 19. August 2020. 
sorgfältige Prüfung der Herkunft durch die Redaktion nicht ersetzen, sondern sollte sie - mit Blick auf eine zumindest potenziell mündige Nutzer*innenschaft - von vornherein ergänzen. Wenn die Nutzenden gewissermaßen »a priori« wissen, dass das Ausgangsmaterial zum Beispiel von ukrainischen Behörden stammt, dann können sie diese Tatsache aktiv zur eigenständigen Einordnung der Behauptung verwenden. Umgangssprachlich gesagt: Dann kann ich als Nutzer die Behauptung eher "glauben« oder ihr, besser gesagt, vertrauen - oder eben meine Zweifel daran haben.

Bernard Pörksen (2018) fragt zukunftsweisend: Wohin entwickeln sich Redaktionen? Oder anders formuliert: Kann »Redaktion « auf neue Weise sogar zu einem gesellschaftlichen Prinzip werden? Er schlägt eine »Utopie der redaktionellen Gesellschaft« vor. Ihm gehe es darum, publizistische Verantwortung neu zu denken und »die Verantwortungszone« auszuweiten. Damit soll eine redaktionelle Gesellschaft das längst medienmächtig gewordene Publikum ähnlich einbeziehen wie den etablierten Journalismus oder auch die Plattformbetreiber. Allerdings erscheint dieser Ansatz zu stark auf Moral und Ethik konzentriert: Pörksen fragt nicht in ausreichendem Maß nach (handfesten) Interessen, Konflikten und Krisen in Gesellschaften wie der gegenwärtigen hierzulande, die er als »liberale Demokratien« bestimmt, womit er zum Beispiel Turbo-Kapitalismus und verschärfte Konkurrenz ignoriert.

Wenn auch das »Handwerkszeug « laut Pörksen bereits vorhanden ist, bleibt eine Aufgabe: Mit Blick auf ihre Publika müssten sich Journalist*innen von ihren bisherigen elitären Höhen in Bewegung setzen »sie müssten sich von der Rolle des Predigers, des Pädagogen und des autoritär auftretenden Wahrheitsverkünders verabschieden« (ebd.). Pörksen versucht, sein Ideal eines transparent und dialogisch orientierten Journalismus anhand von zwei Imperativen zu formulieren: Erstens gebe man seinem Publikum »jede nur denkbare Möglichkeit, die Qualität der von dir vermittelten Informationen einzuschätzen!«. Auch in dieser Hinsicht scheint frühestmögliche Transparenz mit Blick auf die Quellenlage eine enorm wichtige »denkbare Möglichkeit«. Und zweitens begreife man die eigene Kommunikation nie als Endpunkt, sondern immer als »Anfang und Anstoß von Dialog und Diskurs«. Auch hier kommt die Quellenfrage zuvörderst ins Spiel - Nutzer*innen können bei bestmöglicher Transparenz weitere Quellen suchen und kritisch einbeziehen und nicht zuletzt den primär Beitragenden als selbst auch Beitragende durch Feedback produktive Anstöße geben (vgl. Köhler 2018). 
Mit Blick auf Transformationsfragen für den Journalismus sind also auch daher Schritte in Richtung des (Wieder-)Gewinnens von Vertrauen in professionelle Medienangebote möglich: Wenn Nutzer*innen möglichst frühzeitig erfahren, wessen Perspektive ihnen hier warum präsentiert wird, dann sollten Chancen für symmetrische(-re) Kommunikation tendenziell steigen (vgl. Phillips 2010, 2-4). Derart könnte sich die Umsetzung der zur Erfüllung von kulturell-transformativen Aufgaben öffentlicher Medien und für weitere Demokratisierungen wichtigen Perspektivenwechsel (vgl. Krüger 1992, 220-229) wenigstens nicht noch weiter erschweren.

Nicht nur Redaktionen und Journalist*innen, sondern auch Publika und Gesellschaft sollten journalistische Selbsttransparenz nutzen. Quellentransparenz als Aspekt der Beitragstransparenz kann auf der Objektebene Nutzer*innen-Aktivität sowie -Vertrauen steigern und Redaktionen, die regelmäßig öffentlich über ihre Arbeit berichten, stoßen sich an und werden angestoßen auf der Metaebene zu mehr Reflexion gerade mit Blick auf ihre öffentliche Aufgabe und Verantwortung (vgl. Reimer 2017, 107-108). Oder in Phillips (2010, 8) Worten: Entwicklungen in Richtung von mehr Transparenz gerade hinsichtlich der Quellen journalistischer Arbeit mögen zu weiteren Demokratisierungen beitragen.

So sollte nicht zuletzt mehr und bessere Quellentransparenz - als ein Vermittlungsglied zwischen Journalist*innen und Nutzer*innen einerseits und gesellschaftlichen Öffentlichkeiten andererseits - Perspektivenwechsel und symmmetrische(-re) Kommunikation eher befördern und progressive Transformationen (in diesem Beitrag skizziert an Beispielen im Kontext friedlichen Zusammenlebens) für möglichst alle Menschen zumindest tendenziell erleichtern - global gesehen und auch für künftige Generationen.

\section{Literatur}

Bilger, Oliver. 2018. Der Mord an Arkadi Babtschenko verdeutlicht die russischukrainische Feindschaft. Tagesspiegel vom 30. Mai, https://www.tagesspiegel. de/politik/toter-kremlkritiker-der-mord-an-arkadi-babtschenko-verdeutlichtdie-russisch-ukrainische-feindschaft/22623110.html. Zugegriffen: 13. November 2020.

Garton Ash, Timothy. 2016. Free Speech. Ten Principles for a Connected World. Redefreiheit. Prinzipien für eine vernetzte Welt. New Haven, London: Yale University Press.

Gurk, Christoph. 2019. Bolivien: »Bürgerkrieg jetzt!« Süddeutsche Zeitung vom 19. 
November, https://www.sueddeutsche.de/politik/bolivien-morales-1.4678557. Zugegriffen: 13. November 2020.

Haller, Michael. 2008. Recherchieren. 7. Auflage. Konstanz. UVK.

Haller, Michael. 2017. Vorwort. In: Öffentliches Vertrauen in der Mediengesellschaft, herausgegeben von Michael Haller, 9-12. Köln: Herbert von Halem.

Köhler, Sebastian. 2015. Aufgehobener Journalismus. Zu einigen Aktualisierungen von Internetnutzungspotenzialen für gelingende gesellschaftliche Kommunikation. In: Gesellschaft. Medien. Rezeption. Wie unsere Wirklichkeit kommunikativ gestaltet wird, herausgegeben von Frank Überall und Klaus-Dieter Schulz, 1332. Münster: Lit.

Köhler, Sebastian. 2018. Mehr beitragen, weniger senden. Zur Rolle von Feedback und Artikulation im Journalismus. Journalistik. Zeitschrift für Journalismusforschung, 1 (2): 51-60. http://journalistik.online/ausgabe-02-2018/mehr-beitragen-weniger-senden/. Zugegriffen: 23. August 2018.

Köhler, Sebastian. 2019. Sieh an - (was für) eine Geschichte! In: Richtung nachhaltiger Narrativität: Zum journalistisch-audiovisuellen Storytelling in Zeiten von »Fake News«. In: Storytelling in Journalismus, Organisations- und Marketingkommunikation, herausgegeben von Silvia Ettl-Huber, 73-98. Wiesbaden: Springer VS.

Krüger, Hans-Peter. 1992. Zur kulturellen Aufgabe öffentlicher Medien in einer modernen Demokratie. In: Demission der Helden. Kritiken von innen 1983-1992, herausgegeben von Hans-Peter Krüger, 220-229. Berlin: Aufbau-Verlag.

Krüger, Uwe, und Michael Meyen. 2018. Auf dem Weg in die Postwachstumsgesellschaft. Plädoyer für eine transformative Kommunikationswissenschaft. Publizistik, 63 (3): 341-357.

Krüger, Uwe. 2020. Buchbesprechung zu Wintterlin, Florian: Quelle: Internet. Journalistisches Vertrauen bei der Recherche in sozialen Medien. Publizistik 65 (2), 291-293. DOI: https://doi.org/10.1007/s11616-020-00570-y.

Meyen, Michael. 2018. Fake Debate: Wem »Fake News« wirklich helfen. In: Medienrealität 2018, herausgegeben von Michael Meyen. https://medienblog.hypotheses.org/1009. Zugegriffen 11. April 2018.

Nordquist, Richard. 2019. The 5 Ws (and an H) of Journalism. https://www. thoughtco.com/journalists-questions-5-ws-and-h-1691205. Zugegriffen: 7. August 2019.

Phillips, Angela. 2010. Transparency and the new Ethics of Journalism. Journalism Practice, 4 (3), 373-382. DOI: 10.1080/17512781003642972.

Phillips, Angela. 2011. Journalists as unwilling »sources«. Transparency and the new ethics of journalism. In: Journalists, Sources, and Credibility. New Perspectives, herausgegeben von Bob Franklin und Matt Carlson, 49-60. New York, London: Routledge.

Pörksen, Bernhard. 2018. Alle müssen Journalisten sein. Die Zeit Nr. 8 vom 15. Februar, http://www.zeit.de/2018/08/umgang-medien-fake-news-propagandajournalismus/komplettansicht. Zugegriffen: 13. November 2020.

Prochazka, Fabian. 2020. Vertrauen in Journalismus unter Online-Bedingungen. Zum Einfluss von Personenmerkmalen, Qualitätswahrnehmungen und Nachrich- 
tennutzung. https://link.springer.com/book/10.1007\%2F978-3-658-30227-6. Wiesbaden: Springer VS.

Reimer, Julius. 2017. Vertrauen durch Transparenz? Potentiale und Probleme journalistischer Selbstoffenbarung. In: Öffentliches Vertrauen in der Mediengesellschaft, herausgegben von Michael Haller, 101-119. Köln: Herbert von Halem.

Rezo. 2020. Was ist guter Journalismus? Die Zeit vom 5. Mai, https://www.zeit.de/ kultur/2020-05/henri-nannen-preis-journalismus-youtube-medien/seite-2. Zugegriffen: 18. Oktober 2020.

Rosa, Hartmut. 2013. Beschleunigung und Entfremdung. Entwurf einer Kritischen Theorie spätmoderner Zeitlichkeit. Berlin: Suhrkamp.

Rudolph, Steffen. 2019. Digitale Medien, Partizipation und Ungleichheit. Eine Studie zum sozialen Gebrauch des Internets. Wiesbaden: Springer VS.

Schultz, Tanjev. 2019. Quelle. In: Journalistikon. Das Wörterbuch der Journalistik. http://journalistikon.de/quelle/. Zugegriffen: 16. November 2019.

Schultz, Tanjev, Marc Ziegerle, Ilka Jakobs, Nikolaus Jackob, Oliver Quiring, und Christian Schemer. 2020. Medienzynismus weiterhin verbreitet, aber mehr Menschen widersprechen. Mainzer Langzeitstudie Medienvertrauen 2019. Media Perspektiven 6: 322-330. https://www.ard-werbung.de/fileadmin/user_upload/media-perspektiven/pdf/2020/0620_Schultz_Ziegele_Jakobs_Jackob_ Quiring_Schemer.pdf. Zugegriffen: 13. November 2020.

vks/dpa. 2018. Arkadij Babtschenko: Die vielen Rätsel um das angebliche Mordfoto. Spiegel Online vom 1. Juni, https://www.spiegel.de/politik/ausland/arkadi-babtschenko-ukraine-wie-das-bild-seiner-leiche-zustandekam-a-1210575.html. Zugegriffen: 13. November 2020.

Waisbord, Silvio. 2019. The 5Ws and $1 \mathrm{H}$ of Digital Journalism. Digital Journalism 7 (3): 351-358. DOI: https://doi.org/10.1080/21670811.2018.1545592.

Weischenberg, Siegfried. 2005. Nachricht. In: Handbuch Journalismus und Medien, herausgegeben von Siegfried Weischenberg, Hans J. Kleinsteuber und Bernhard Pörksen, 306-308. Konstanz: UVK.

Wintterlin, Florian. 2020. Quelle: Internet. Journalistisches Vertrauen bei der Recherche in sozialen Medien. Baden-Baden: Nomos.

Wolff, Volker, und Carla Palm. 2006a. Bericht. In: Lexikon Kommunikations- und Medienwissenschaft, herausgegeben von Günter Bentele, Hans-Bernd Brosius und Otfried Jarren, 21-22. Wiesbaden: VS Verlag für Sozialwissenschaften.

Wolff, Volker, und Carla Palm. 2006b. Meldung. In: Lexikon Kommunikations- und Medienwissenschaft, herausgegeben von Günter Bentele, Hans-Bernd Brosius und Otfried Jarren, 190. Wiesbaden: VS Verlag für Sozialwissenschaften.

\section{Open Access}

Dieser Beitrag erscheint unter der Creative-Commons-Lizenz CC BY-ND 3.0 DE: https://creativecommons.org/licenses/by-nd/3.0/de/. 